\title{
Computing the Pluricomplex Green Function with Two Poles
}

\author{
Frank Wikström
}

\section{CONTENTS}

1. Introduction

2. Computing $\delta$

3. Computing $\delta^{*}$

4. Numerical Computation of $\delta$

5. Numerical Computation of $\delta^{*}$

6. Results of the Numerical Computation

7. A Counterexample to Coman's Conjecture

Acknowledgments

References

2000 AMS Subject Classification: Primary 32U35; Secondary 32F45

Keywords: Pluricomplex Green function, Lempert function, interval arithmetic
We look at numerical computations of the pluricomplex Green function $g$ with two poles of equal weight for the bidisk. The results we obtain strongly suggest that Coman's conjecture holds in this setting, that is that $g$ equals the Lempert function. We also prove this in a special case.

Furthermore, we show that Coman's conjecture fails in the case of two poles of different weight in the unit ball of $\mathbb{C}^{2}$.

\section{INTRODUCTION}

Plurisubharmonic functions play an important role in the study of holomorphic functions of several variables in much the same way the subharmonic functions do in one complex variable. Recall that a function $u$ defined on some open set $\Omega$ in $\mathbb{C}^{n}$ is said to be plurisubharmonic if $u$ is upper semicontinuous and if for every $z_{0} \in \Omega$ and every $w \in \mathbb{C}^{n}$, the function $\mathbb{C} \ni \zeta \mapsto u\left(z_{0}+\zeta w\right)$ is subharmonic (where it is defined). PluriPotential Theory (PPT) is, loosely speaking, the study of plurisubharmonic functions, and has grown to become an independent area of research. In many cases, one is interested in finding out which concepts from Classical Potential Theory (CPT) carry over to the plurisubharmonic setting and which do not. Often, there are strong similarities between CPT and PPT, but in many cases the theorems in pluripotential theory require new proofs, since many of the techniques available in CPT are no longer there for us to use in higher dimensions. The most notable difference is perhaps that CPT focuses around properties of the Laplace operator, $\Delta$, which of course is a very well-studied and well-understood operator. The differential operator which lies at the heart of PPT is the complex Monge-Ampère operator, MA, a fully nonlinear second order differential operator. The nonlinearity of MA makes things much more complicated - it is not even possible to define $\mathrm{MA}(u)$ (in a reasonable way) for every plurisubharmonic function $u$. This is in sharp contrast

(c) A K Peters, Ltd $1058-6458 / 2003 \$ 0.50$ per page Experimental Mathematics 12:3, page 375 
with CPT, since $\Delta$ is a linear operator and therefore $\Delta u$ makes sense for every distribution $u$.

If $u$ is plurisubharmonic and $C^{2}$, we define

$$
\operatorname{MA}(u)=4^{n} n ! \operatorname{det}\left(\frac{\partial^{2} u}{\partial z_{j} \partial \bar{z}_{k}}\right) \mathrm{d} V
$$

as (some constant times) the determinant of the complex Hessian of $u$ times the (2n-dimensional) Lebesgue measure. A considerable amount of work has been devoted to extending this definition to a larger class of plurisubharmonic functions. It is reasonable to say that the advent of pluripotential theory was the very influential 1976 paper by Bedford and Taylor [Bedford and Taylor 76] where they defined MA for locally bounded plurisubharmonic functions. Since then, a number of authors have extended this definition to cover various classes of unbounded plurisubharmonic functions. In particular, we want to mention the paper by Demailly [Demailly 87] where it was shown that there is a natural extension of Bedford and Taylor's definition to allow for plurisubharmonic functions with (a finite number of) logarithmic poles. We refer to the monograph by Klimek [Klimek 91] and the more recent survey by Błocki [Błocki 99] for a more complete picture.

In this paper, we will be concerned with the pluricomplex Green function. In many ways, this function behaves like the Green function for the Laplace operator, but there are notable differences.

Definition 1.1. Let $u$ be a plurisubharmonic function defined on some neighborhood of $w$. Let $\nu>0$. We say that $u$ has a logarithmic pole of order at least $\nu$ at $w$ if there is a constant $c$ such that $u(z) \leq \nu \log |z-w|+c$ for $z$ sufficiently close to $w$. We define the Lelong number of $u$ at $w, \nu_{u}(w)$, as the supremum of all $\nu$ such that $u$ has a logarithmic pole of order at least $\nu$ at $w$.

Definition 1.2. Let $\Omega$ be a domain in $\mathbb{C}^{n}$. If $\nu: \Omega \rightarrow \mathbb{R}_{+}$ is a non-negative function on $\Omega$ such that $\operatorname{supp} \nu$ is finite, then we say that $\nu$ is admissible.

For simplicity of notation, if $p \in \Omega$, we identify $p$ with the characteristic function of $\{p\}$. With these preliminaries at hand, we can define the pluricomplex Green function.

Definition 1.3. Let $\Omega$ be a domain in $\mathbb{C}^{n}$, and let $\nu$ be an admissible function on $\Omega$. The pluricomplex Green function with poles defined by $\nu, g(z ; \nu)$ is defined by

$$
g(z ; \nu)=\sup \left\{u(z): u \in \mathcal{P S} \mathcal{H}(\Omega), u \leq 0, \nu_{u} \geq \nu\right\} .
$$

If we want to emphasize the dependence on $\Omega$, we sometimes write $g_{\Omega}$.

If $\Omega$ is bounded, it is not difficult to check that $g(z ; \nu)$ is a member of its defining class, i.e., that $g(z ; \nu)$ is negative plurisubharmonic and $\nu_{g} \geq \nu$. In fact, we even have that $\nu_{g}=\nu$. In a sense, $g(\cdot ; \nu)$ is a fundamental solution for MA-more precisely $\operatorname{MA}(g(\cdot ; \nu))=$ $\sum_{w \in \Omega}(2 \pi \nu(w))^{n} \delta_{w}$ (note that the sum is finite). This definition of the pluricomplex Green function is due to Lelong [Lelong 89], but in the singleton case, it goes back to Klimek [Klimek 85]. For the basic properties of these functions, such as continuity, we refer to [Demailly 87] and [Lelong 89].

It is a remarkable fact that the single pole Green function in convex domains can be constructed by using "simple one-variable" techniques. More precisely, let $\Omega$ be a bounded domain and let $\nu$ be an admissible function on $\Omega$. Consider the pluricomplex Green function $g_{\Omega}(\cdot)=$ $g_{\Omega}(\cdot ; \nu)$ with poles defined by $\nu=\nu_{1} w_{1}+\cdots+\nu_{p} w_{p}$. Let $\phi: \mathbb{D} \rightarrow \Omega$ be an analytic disk in $\Omega$, with $\phi(0)=z$, and $\phi(\mathbb{D}) \supset \operatorname{supp} \nu$. Assume that $\phi\left(\zeta_{j}\right)=w_{j}$. Then $u=g_{\Omega} \circ \phi$ is a negative subharmonic function on the unit disk in $\mathbb{C}$ and one can verify that $\nu_{u}\left(\zeta_{j}\right) \geq \nu_{g}(\phi(\zeta))=\nu\left(w_{j}\right)$. Thus, if we define $\tilde{\nu}: \mathbb{D} \rightarrow \mathbb{R}_{+}$by $\tilde{\nu}=\nu_{1} \zeta_{1}+\cdots+\nu_{p} \zeta_{p}$, then $\tilde{\nu}$ is an admissible function on $\mathbb{D}$ and $g_{\mathbb{D}}(\zeta ; \tilde{\nu}) \geq u(\zeta)$. Using the linearity of the Laplace operator, one checks that

$$
g_{\mathbb{D}}(\zeta ; \tilde{\nu})=\sum_{j=1}^{p} \nu_{j} \log \left|\frac{\zeta-\zeta_{j}}{1-\bar{\zeta}_{j} \zeta}\right| .
$$

In other words, we see that $u(0)=g_{\Omega}(z ; \nu) \leq g_{\mathbb{D}}(0 ; \tilde{\nu})=$ $\sum \nu_{j} \log \left|\zeta_{j}\right|$. Motivated by this discussion, we make the following definition.

Definition 1.4. Let $\Omega$ be a domain in $\mathbb{C}^{n}$ and let $\nu$ be an admissible function on $\Omega$. Let $\phi: \mathbb{D} \rightarrow \Omega$ be an analytic disk. We say that $\phi$ is $\nu$-admissible if $\phi(\mathbb{D}) \supset \operatorname{supp} \nu$. If $\phi$ is $\nu$-admissible, we define

$$
d(\phi)=\sum_{w \in \operatorname{supp} \nu} \inf \left\{\nu(w) \log |\zeta|: \zeta \in \phi^{-1}(w)\right\} .
$$

Finally, we define the Lempert function with pole defined by $\nu$ by

$$
\delta(z ; \nu)=\inf \{d(\phi): \phi(0)=z, \phi \text { is a } \nu \text {-admissible disk }\} .
$$

From the discussion preceding the definition of $\delta$, we see that if $\nu$ is admissible, then $g(z ; \nu) \leq \delta(z ; \nu)$, and it is straightforward to verify that $g(\cdot ; \nu)=\delta(\cdot ; \nu)$ if $\delta$ is 
plurisubharmonic. The main reason for introducing the Lempert function is that in many ways, it is easier to study $\delta$ than to study $g$, and using $\delta$, we can at least get an upper bound for $g$. Let us move on and define another useful function that will give us a lower bound for $g$.

Definition 1.5. Let $\Omega$ be a domain in $\mathbb{C}^{n}$ and let $\nu$ be an integer-valued admissible function on $\Omega$. Define the Carathéodory function with poles defined by $\nu$ as

$$
\delta^{*}(z ; \nu)=\sup \left\{\log |f(z)|: f \in \mathcal{O}(\Omega, \mathbb{D}), \nu_{\log |f|} \geq \nu\right\} .
$$

The reason for restricting ourselves to integer-valued admissible functions is that if $f$ is holomorphic, then the Lelong number of $\log |f|$ is integer-valued.

Clearly, $\delta^{*}(z ; \nu) \leq g(z ; \nu)$, since the defining family for $\delta^{*}$ is a proper subset of the defining family for $g$. As in the case of $\delta$, the main reason for introducing $\delta^{*}$ is that in some cases, it is easier to estimate $\delta^{*}$ than to estimate $g$ directly.

Furthermore, if $\nu=w$, then $\delta(z ; w)$ and $\delta^{*}(z ; w)$ are closely related to the Kobayashi and Carathéodory pseudodistances, respectively. A deep and very influential theorem by Lempert [Lempert 81] shows that these pseudodistances coincide in convex domains, so we have the following important result:

Theorem 1.6. (Lempert.) Let $\Omega$ be a convex domain, and let $w \in \Omega$. Then $g(z ; w)=\delta(z ; w)=\delta^{*}(z ; w)$.

As a consequence of this theorem, we see that $g(z ; w)=g(w ; z)$ for convex domains, something that is not true in general. (Compare this with the Green function for the Laplace operator which is always symmetric.)

Let us move on to the general case, where $\operatorname{supp} \nu$ is finite but not (necessarily) a singleton. Here, few results are known. Coman [Coman 00] has computed $g(z ; \nu)$ for the unit ball $\mathbb{B}$ in $\mathbb{C}^{n}$ in the case when $\nu=w_{1}+w_{2}$. In fact, Coman computed $\delta(z ; \nu)$ and showed that this function is plurisubharmonic on $\mathbb{B}$, and hence that $\delta=g$ in this particular case. An independent computation by Edigarian and Zwonek [Edigarian and Zwonek 98] used a branched covering map from $\mathbb{B}$ to $\mathcal{E}(1 / 2,1)=\{(z, w) \in$ $\left.\mathbb{C}^{2}:|z|+|w|^{2}<1\right\}$, mapping both poles in $\mathbb{B}$ to the same point in $\mathcal{E}(1 / 2,1)$, to come up with the same result.

Seeing that $g=\delta$, at least in the special case mentioned above, led Coman [Coman 00] to pose the following conjecture.
Conjecture 1.7. Let $\Omega$ be a bounded convex domain in $\mathbb{C}^{n}$, and let $\nu$ be an admissible function on $\Omega$. Then $\delta(z ; \nu)=g(z ; \nu)$.

One other explicit example is for the bidisk $\mathbb{D}^{2}$, when $\nu=w_{1}+w_{2}$, and $w_{1}, w_{2} \in \mathbb{D} \times\{0\}$. For this particular case, an explicit formula for $g(z ; \nu)$ was given by Carlehed [Carlehed 95]. (See also Edigarian and Zwonek [Edigarian and Zwonek 98] for an alternative proof.)

In both of the above cases, one can verify that $\delta=$ $g=\delta^{*}$ and one is tempted to pose a generalization of Coman's conjecture, i.e., that $\delta=g=\delta^{*}$ for convex domains and integer-valued admissible functions. Unfortunately, Coman's conjecture is not true in general. Recently, Carlehed and Wiegerinck [Carlehed and Wiegerinck 03] extended the example in the bidisk to the case $w_{1}, w_{2} \in \mathbb{D} \times\{0\}$ and $\nu\left(w_{1}\right) \neq \nu\left(w_{2}\right)$ for which they compute $g$ and show that $g \neq \delta$.

The question now is whether Coman's conjecture is true under some stronger assumptions, for example, that all poles have the same weight, or if the two positive cases known are purely coincidental. Both of these cases have a high symmetry, and in fact, both of them can be explained by looking at branched covering maps, in case of the bidisk, a double covering from $\mathbb{D}^{2}$ to itself. In view of this, it is natural to ask for a positive result in a less symmetric position of the poles. It is possible to show that for a generic position of two poles in the bidisk, there is no branched covering map from $\mathbb{D}^{2}$ to itself, mapping both poles to the same point. In this paper, we study the case of two poles of equal weight in the bidisk, when there is no restriction on the placement of the poles. It seems difficult to obtain an explicit formula for $g$ (or $\delta$ or $\left.\delta^{*}\right)$ in this general setting, but we show how these functions can be computed numerically. The results obtained from the numerical calculations strongly suggest that $\delta=g=\delta^{*}$ in the case of two poles of equal weight in the bidisk.

In the final section of the paper, we show that Coman's conjecture fails in the unit ball of $\mathbb{C}^{2}$. More precisely, if $\nu=w+2 w^{\prime}$, where $w=0$ and $w^{\prime}=(1 / 2,0)$, then $\delta(\cdot ; \nu)$ is not plurisubharmonic and hence $g(\cdot ; \nu) \neq \delta(\cdot ; \nu)$. This counterexample is done using interval arithmetic.

During the preparation of this paper, I learned that Pascal Thomas and Nguyen Van Trao [Thomas and Trao 03] have shown that if $\Omega=\mathbb{D}^{2}$ and $\nu=p+p^{\prime}+q+q^{\prime}$ where $p=(a, 0) ; p^{\prime}=(a, \varepsilon), q=(-a, 0)$, and $q^{\prime}=(-a, \varepsilon)$ where $a \in \mathbb{D} ;$ and $|\varepsilon|$ is small enough, then $\delta(z ; \nu) \neq$ $g(z ; \nu)$. 


\section{COMPUTING $\delta$}

Let $p, q \in \mathbb{D}^{2}$ and $\nu=p+q$. We want to compute $\delta(z ; \nu)$. It is clear that $\delta$ is biholomorphically invariant, so we may as well assume that $z=0$. Take any $\nu$-admissible disk $\phi$ with $\phi(0)=0$, i.e., $\phi$ is an analytic disk with $\phi(\alpha)=p$ and $\phi(\beta)=q$ for some $\alpha$ and $\beta$ in $\mathbb{D}$. Write $\phi=\left(\phi_{1}, \phi_{2}\right)$ for the components of $\phi$. Then for each $j$ $(j=1,2)$, we have a holomorphic function $\phi_{j}: \mathbb{D} \rightarrow$ $\mathbb{D}$ such that $\phi_{j}(0)=0, \phi_{j}(\alpha)=p_{j}$, and $\phi_{j}(\beta)=q_{j}$. By the Nevanlinna-Pick interpolation theorem [Pick 16], there exists $\phi_{j}$ with these properties if and only if the Nevanlinna-Pick matrix

$$
P_{j}=\left(\begin{array}{ccc}
1 & 1 & 1 \\
1 & \frac{1-\left|p_{j}\right|^{2}}{1-\left|\alpha^{2}\right|} & \frac{1-p_{j} \bar{q}_{j}}{1-\alpha \bar{\beta}} \\
1 & \frac{1-\bar{p}_{j} q_{j}}{1-\bar{\alpha} \beta} & \frac{1-\left|q_{j}\right|^{2}}{1-|\beta|^{2}}
\end{array}\right)
$$

is positive semidefinite. Hence, $\delta(0 ; \nu)=\inf \{\log |\alpha|+$ $\left.\log |\beta|: P_{1}, P_{2} \geq 0\right\}$. For ease of computation, let $\tilde{\delta}=$ $\exp \delta=\inf \left\{|\alpha \beta|: P_{1}, P_{2} \geq 0\right\}$. Expanding the minors of $P_{j}$, we see that $P_{1}$ and $P_{2}$ are positive semidefinite if and only if

$$
\begin{aligned}
& |\alpha| \geq \max \left\{\left|p_{1}\right|,\left|p_{2}\right|\right\}, \\
& |\beta| \geq \max \left\{\left|q_{1}\right|,\left|q_{2}\right|\right\},
\end{aligned}
$$

and

$$
\left|\frac{\alpha-\beta}{1-\alpha \bar{\beta}}\right| \geq \max \left\{\left|\frac{p_{1} \beta-q_{1} \alpha}{\alpha \bar{\beta}-p_{1} \bar{q}_{1}}\right|,\left|\frac{p_{2} \beta-q_{2} \alpha}{\alpha \bar{\beta}-p_{2} \bar{q}_{2}}\right|\right\} .
$$

In other words, we want to minimize $|\alpha \beta|$ under the conditions $(2-1),(2-2)$, and $(2-3)$. For a generic choice of $p$ and $q$, this turns out to be a very messy computation, but let us attack the special case $p=(p, 0), q=(0, q)$.

Proposition 2.1. Let $p=(p, 0), q=(0, q)$, and $\nu=p+q$. Then

$$
\delta(0 ; \nu)=\log \frac{|p q|}{|p|+|q|-|p q|} .
$$

Proof: Let $\phi: \mathbb{D} \rightarrow \mathbb{D}^{2}$ be a $\nu$-admissible disk with $\phi(0)=$ $0, \phi(\alpha)=p$, and $\phi(\beta)=q$. Let us choose $d \in \mathbb{R}_{+}$ and $\theta \in \mathbb{R}$ such that $\alpha \bar{\beta}=d e^{i \theta}$. When $p_{2}=q_{1}=0$, Equations (2-1)-(2-3) reduce to

$$
\begin{gathered}
|\alpha| \geq|p|, \\
|\beta| \geq|q|, \\
\left|\frac{\alpha-\beta}{1-\alpha \bar{\beta}}\right| \geq\left|\frac{p}{\alpha}\right|,
\end{gathered}
$$

and

$$
\left|\frac{\alpha-\beta}{1-\alpha \bar{\beta}}\right| \geq\left|\frac{q}{\beta}\right| .
$$

Squaring (2-7) and (2-8) and rearranging the results, we obtain the following inequalities:

$$
\begin{aligned}
|p|^{2}\left(1-2 d \cos \theta+d^{2}\right) & \leq|\alpha|^{2}\left(|\alpha|^{2}+|\beta|^{2}-2 d \cos \theta\right) \\
& =|\alpha|^{4}+d^{2}-2 d|\alpha|^{2} \cos \theta,
\end{aligned}
$$

and

$$
\begin{aligned}
|q|^{2}\left(1-2 d \cos \theta+d^{2}\right) & \leq|\beta|^{2}\left(|\alpha|^{2}+|\beta|^{2}-2 d \cos \theta\right) \\
& =d^{2}+|\beta|^{4}-2 d|\beta|^{2} \cos \theta,
\end{aligned}
$$

respectively. Furthermore, from (2-5) and (2-6), using that $|\alpha \beta|=d$, we have that

$$
|p|^{2} \leq|\alpha|^{2} \leq \frac{d^{2}}{|q|^{2}}
$$

Hence, we want to find the smallest value of $d$, such that $(2-9)$ and $(2-10)$ are satisfied for some value of $\theta$ with the additional constraint $(2-11)$.

Rearranging (2-9) and (2-10) temporarily, we have that

$$
2 d \cos \theta\left(|\alpha|^{2}-|p|^{2}\right) \leq|\alpha|^{4}+d^{2}-|p|^{2}-|p|^{2} d^{2}
$$

and

$$
2 d \cos \theta\left(|\beta|^{2}-|q|^{2}\right) \leq|\beta|^{4}+d^{2}-|q|^{2}-|q|^{2} d^{2} .
$$

From this, we note that if (2-9) and (2-10) are satisfied for some $\left(d, \alpha, \theta_{0}\right)$, they are satisfied for every $(d, \alpha, \theta)$, such that $\cos \theta \leq \cos \theta_{0}$. Hence, we may as well assume that $\theta=\pi$. Using this observation, we rewrite $(2-9)$ and $(2-10)$ once again, obtaining

$$
|p|^{2}(1+d)^{2} \leq\left(|\alpha|^{2}+d\right)^{2}
$$

and

$$
|q|^{2}(1+d)^{2} \leq\left(|\beta|^{2}+d\right)^{2} .
$$

Taking square roots and rearranging yet again, we end up with the two inequalities:

$$
|p|(1+d)-d \leq|\alpha|^{2},
$$

and

$$
|q|(1+d)-d \leq|\beta|^{2} .
$$

Now, since $\delta(z ; p+q) \leq \min \{\delta(z ; p), \delta(z ; q)\}$ (see [Wikström 99]), we may assume that $d \leq \min \{|p|,|q|\}$, and hence that $|p|(1+d)-d \geq|p|^{2}$ and $|q|(1+d)-d \geq$ 
$|q|^{2}$. In particular, we may assume that the left-hand sides of $(2-14)$ and $(2-15)$ are both positive. Multiplying (2-14) and (2-15), we obtain

$$
|p||q|(1+d)^{2}+d^{2}-d(1+d)(|p|+|q|) \leq d^{2} .
$$

Solving for $d$ in $(2-16)$ gives

$$
d \geq d_{0}:=\frac{|p q|}{|p|+|q|-|p q|} .
$$

Conversely, by taking

$$
\alpha=\sqrt{|p|\left(1+d_{0}\right)-d_{0}}=\frac{|p|}{\sqrt{|p|+|q|-|p q|}}
$$

and

$$
\beta=-\sqrt{|q|\left(1+d_{0}\right)-d_{0}}=-\frac{|q|}{\sqrt{|p|+|q|-|p q|}},
$$

a routine calculation shows that conditions through (2-8) are satisfied. Hence,

$$
\delta(0 ; \nu)=\log \frac{|p q|}{|p|+|q|-|p q|},
$$

as claimed.

For $p$ and $q$ in general position, we will resort to numerical computations.

\section{COMPUTING $\delta^{*}$}

To compute $\delta^{*}$, we are naturally led to study certain interpolation problems for holomorphic functions. The classical result for the unit disc in this direction is the Nevanlinna-Pick theorem [Pick 16], which we already used in Section 2..

Theorem 3.1. Let $\left(w_{1}, \ldots, w_{n}\right) \in \mathbb{D}^{n}$. Then there is a function $f \in H^{\infty}(\mathbb{D})$ with $\|f\| \leq 1$, such that $f\left(\alpha_{j}\right)=w_{j}$, $1 \leq j \leq n$ if and only if the matrix

$$
\left(\frac{1-w_{j} \bar{w}_{k}}{1-\alpha_{j} \bar{\alpha}_{k}}\right)_{i, j=1}^{n}
$$

is positive semidefinite.

Recently, Agler [Agler 98] gave a generalization of the Nevanlinna-Pick interpolation theorem to the bidisk. For a published proof of a more general result, see [Agler and McCarthy 99].

Theorem 3.2. (Agler.) Let $\left(w_{1}, \ldots, w_{n}\right) \in \mathbb{D}^{n}$. Then there is a $f \in H^{\infty}\left(\mathbb{D}^{2}\right)$, with $\|f\| \leq 1$ such that $f\left(\lambda_{1}^{j}, \lambda_{2}^{j}\right)=w_{j}, 1 \leq j \leq n$ if and only if there are positive semidefinite $n \times n$-matrices $A$ and $B$ such that

$1-w_{j} \bar{w}_{k}=\left(1-\lambda_{1}^{j} \bar{\lambda}_{1}^{k}\right) A_{j k}+\left(1-\lambda_{2}^{j} \bar{\lambda}_{2}^{k}\right) B_{j k}, \quad 1 \leq j, k \leq n$.

To compute $\delta^{*}(0 ; \nu)$, where $\nu=p+q$, we look at functions $f \in \mathcal{O}\left(\mathbb{D}^{2}, \mathbb{D}\right)$ such that $f(p)=f(q)=0$ and $|f(0)|$ is as large as possible. In other words, we want to solve the following problem: Maximize $|c|$ under the side condition

$$
\left(\begin{array}{ccc}
1-|c|^{2} & 1 & 1 \\
1 & 1 & 1 \\
1 & 1 & 1
\end{array}\right)=P_{1} \otimes A+P_{2} \otimes B
$$

where

$$
P_{j}=\left(\begin{array}{ccc}
1 & 1 & 1 \\
1 & 1-\left|p_{j}\right|^{2} & 1-p_{j} \bar{q}_{j} \\
1 & 1-\bar{p}_{j} q_{j} & 1-\left|q_{j}\right|^{2}
\end{array}\right), \quad j=1,2
$$

for some positive semidefinite matrices $A$ and $B$. Here, $\otimes$ denotes the Schur-product, or element-wise product, i.e., $\left(a_{j k}\right) \otimes\left(b_{j k}\right)=\left(a_{j k} b_{j k}\right)$.

In our setting, it can be proved that it is enough to look for solutions to $(3-2)$ where $A$ and $B$ both have rank 1, but the proof of this is rather tedious and we omit it. For our purposes, the computations below will justify that we only look for rank one solutions.

Again, as for explicitly computing $\delta$, it seems to be a rather difficult task to compute $\delta^{*}$, but we can solve the same special case as we did for $\delta$.

Proposition 3.3. Let $p=(p, 0), q=(0, q)$ and let $\nu=$ $p+q$. Then

$$
\delta^{*}(0 ; \nu)=\log \frac{|p q|}{|p|+|q|-|p q|} .
$$

Proof: Since $\delta^{*}$ is invariant under biholomorphic mappings of $\mathbb{D}^{2}$, we may as well assume that $p$ and $q$ are positive real. Seeking real rank 1 solutions to Equation (3-2), we write $A=x^{\mathrm{T}} x$ and $B=y^{\mathrm{T}} y$, where $x, y \in \mathbb{R}^{3}$. With this approach, Equation $(3-2)$ reduces to $x_{1}^{2}+y_{1}^{2}=1-c^{2}$ and

$$
\begin{cases}x_{1} x_{2}+y_{1} y_{2} & =1 \\ x_{1} x_{3}+y_{1} y_{3} & =1 \\ x_{2} x_{3}+y_{2} y_{3} & =1 \\ \left(1-p^{2}\right) x_{2}^{2}+y_{2}^{2} & =1 \\ x_{3}^{2}+\left(1-q^{2}\right) y_{3}^{2} & =1 .\end{cases}
$$

It turns out that, with a little help from computer algebra, one can solve (3-4) for $x_{1}, x_{2}, x_{3}, y_{1}$, and $y_{2}$ in terms 
of $y_{3}$. Now, maximizing $c$ means minimizing $x_{1}^{2}+y_{1}^{2}$, and if we write this expression in terms of $y_{3}$ alone, this is just an optimization problem for a function of one real variable, which is easily solved, again with some help from Maple. Omitting the details, the result of these computations is that

$$
A=x^{\mathrm{T}} x=\left(\begin{array}{ccc}
\frac{q(1-p)(p+q)}{(p+q-p q)^{2}} & \frac{q}{p+q-p q} & \frac{q(1-p)}{p+q-p q} \\
\frac{q}{p+q-p q} & \frac{q}{(1-p)(p+q)} & \frac{q}{p+q} \\
\frac{q(1-p)}{p+q-p q} & \frac{q}{p+q} & \frac{q(1-p)}{p+q}
\end{array}\right)
$$

and

$$
B=y^{\mathrm{T}} y=\left(\begin{array}{ccc}
\frac{p(1-q)(p+q)}{(p+q-p q)^{2}} & \frac{p(1-q)}{p+q-p q} & \frac{p}{p+q-p q} \\
\frac{p(1-q)}{p+q-p q} & \frac{p(1-q)}{p+q} & \frac{p}{p+q} \\
\frac{p+q}{p+q-p q} & \frac{p}{p+q} & \frac{p}{(1-q)(p+q)}
\end{array}\right)
$$

are solutions to Equation (3-2). Hence,

$$
\begin{aligned}
\delta^{*}(0 ; \nu) & \geq \frac{1}{2} \log \left(1-\frac{q(1-p)(p+q)}{(p+q-p q)^{2}}-\frac{p(1-q)(p+q)}{(p+q-p q)^{2}}\right) \\
& =\frac{1}{2} \log \left(\frac{p^{2} q^{2}}{(p+q-p q)^{2}}\right)=\log \left(\frac{p q}{p+q-p q}\right) .
\end{aligned}
$$

Since $\delta^{*} \leq \delta$, the inequality in $(3-7)$ must in fact be an equality, and the proof is complete.

Corollary 3.4. Let $p=(p, 0), q=(0, q)$ and let $\nu=p+q$. Then

$$
g(0 ; \nu)=\log \frac{|p q|}{|p|+|q|-|p q|} .
$$

\section{NUMERICAL COMPUTATIONS OF $\delta$}

Since the explicit computations of $\delta$ and $\delta^{*}$ in the general case seem difficult to carry out, we turn to numerical computations. Recall that we want to minimize $|\alpha \beta|$ under the conditions

$$
\begin{aligned}
& |\alpha| \geq \max \left\{\left|p_{1}\right|,\left|p_{2}\right|\right\}, \\
& |\beta| \geq \max \left\{\left|q_{1}\right|,\left|q_{2}\right|\right\},
\end{aligned}
$$

and

$$
\left|\frac{\alpha-\beta}{1-\alpha \bar{\beta}}\right| \geq \max \left\{\left|\frac{p_{1} \beta-q_{1} \alpha}{\alpha \bar{\beta}-p_{1} \bar{q}_{1}}\right|,\left|\frac{p_{2} \beta-q_{2} \alpha}{\alpha \bar{\beta}-p_{2} \bar{q}_{2}}\right|\right\} .
$$

After a rotation of $\mathbb{D}$, we may assume that $\alpha$ is real and positive. It turns out that the following simple-minded approach to minimizing $\alpha|\beta|$ works well:
1. Let $\Theta=[0,2 \pi]$.

2. Choose $\theta_{1}, \ldots \theta_{n} \in \Theta$ equidistantly. (By default, $n$ is taken to be 25.)

3. For each $\theta_{j}$, compute $\delta_{j}=\min \alpha|\beta|$ under the side conditions (4-1)-(4-3) and the extra assumption that $\beta=b e^{i \theta_{j}}, 0<b<1$. This minimization, in turn, is done in the following way:

(a) Let $A=\left[\max \left\{\left|p_{1}\right|,\left|p_{2}\right|\right\}, 1\right]$.

(b) Choose $\alpha_{1}, \ldots, \alpha_{m} \in A$ equidistantly. (By default, $m$ is taken to be 20.)

(c) For each $\alpha_{k}$, compute $d_{k}=\alpha_{k} b_{k}$, where $b_{k}$ is the smallest value of $b$ such that $(4-1)-(4-3)$ are fulfilled with $\alpha=\alpha_{k}$ and $\beta=b e^{i \theta_{j}}$. Note that $b_{k}$ can be computed from (4-3) by solving a quartic equation.

(d) Let $A=\left[\alpha_{k_{0}-k^{\prime}}, \alpha_{k_{0}+k^{\prime}}\right]$, where $k_{0}$ is the index such that $d_{k_{0}}=\min _{k} d_{k}$ and $k^{\prime}$ is a suitable small integer. (By default, $k^{\prime}=3$.)

(e) If the diameter of $A$ is small enough (by default, less than $10^{-8}$ ), return $d_{k_{0}}$, otherwise goto Step 3(b).

4. Let $\Theta=\left[\theta_{j_{0}-j^{\prime}}, \theta_{j_{0}+j^{\prime}}\right]$, where $j_{0}$ is the index such that $\delta_{j_{0}}=\min _{j} \delta_{j}$ and $j^{\prime}$ is a suitable small integer. (By default, $j^{\prime}=3$.)

5. If the diameter of $\Theta$ is small enough (by default, less than $\left.10^{-8}\right)$, return $\delta_{j_{0}}$, otherwise goto Step 2 .

In practice, the simple algorithm described above works surprisingly well. If $p$ or $q$ is very close to the boundary, the result of the computation may be a nonglobal minimum of $\alpha|\beta|$, and in that case, the default values of the parameters $m, n, k^{\prime}$, and $j^{\prime}$ in the method may have to be changed.

\section{NUMERICAL COMPUTATIONS OF $\delta^{*}$}

In this section, we look at the methods that were used to compute $\delta^{*}$ numerically. More precisely, let us take $p, q \in \mathbb{D}^{2}$ and try to compute $\delta^{*}(0 ; p+q)$.

As described in Section 3., we want to find the largest possible value of $c$ for which Equation (3-2) has a solution with $A$ and $B$ positive semidefinite. It can be shown that it is enough to consider the case where $A$ and $B$ both are of rank 1. Fix the real number $c>0$. Write $A=\bar{a}^{\mathrm{T}} a$, where $a=\left(a_{1}, a_{2}, a_{3}\right)$. Note that every rank-1 positive semidefinite matrix can be written in this way, with $a$ 
uniquely determined up to a common rotation of all three components. We assume that $a$ is chosen in a way so that $a_{1} \in \mathbb{R}_{+}$. Write $a_{2}=x+i y$ and $a_{3}=u+i v$. In a similar way, we write $B=\bar{b}^{\mathrm{T}} b$, where $b=\left(b_{1}, b_{2}, b_{3}\right)$

Looking at the first column in Equation (3-2), we get

$$
\begin{aligned}
a_{1}^{2}+\left|b_{1}\right|^{2} & =1-c^{2} \\
a_{1} a_{2}+\bar{b}_{1} b_{2} & =1 \\
a_{1} a_{3}+\bar{b}_{1} b_{3} & =1 .
\end{aligned}
$$

By choosing $b_{1}>0$, we see that $b$ is uniquely determined by $a$ from (5-1). The remaining equations in (3-2) then give

$$
\begin{aligned}
\left(1-\left|p_{1}\right|^{2}\right)\left|a_{2}\right|^{2}+\left(1-\left|p_{2}\right|^{2}\right)\left|b_{2}\right|^{2} & =1 \\
\left(1-p_{1} \bar{q}_{1}\right) a_{2} \bar{a}_{3}+\left(1-p_{2} \bar{q}_{2}\right) b_{2} \bar{b}_{3} & =1 \\
\left(1-\left|q_{1}\right|^{2}\right)\left|a_{3}\right|^{2}+\left(1-\left|q_{2}\right|^{2}\right)\left|b_{3}\right|^{2} & =1 .
\end{aligned}
$$

By substituting the values for $b_{1}, b_{2}$, and $b_{3}$ obtained from Equation (5-1) into (5-2) and separating the real and imaginary parts, we get a system of four equations in the five real variables $a_{1}, x, y, u, v$, say $F\left(a_{1}, x, y, u, v\right)=$ 0 , where $F=\left(F_{1}, F_{2}, F_{3}, F_{4}\right)$. The equation $F=0$ is then solved numerically in the following way:

1. Choose a starting value for $a_{1}, x, y, u, v$.

2. Try to minimize the function $\|F\|^{2}$ using a number of iterations with the steepest descent method combined with a line-search. (Note that the derivatives of $F$ can be computed explicitly without too much trouble.)

3. Fix the value of $a_{1}$ obtained from Step 2 and solve $F=0$ for $x, y, u, v$ using Newton's method combined with a line search.

This algorithm is repeated until a solution is found (in the implementation, we require $\|F\|^{2}<10^{-28}$ for a solution) or the maximum number of iterations is reached. If a solution is found, we increase the value of $c$ and start over again. When $p$ or $q$ is very close to the boundary, this method occasionally gives a less than optimal result. In such cases, it was necessary to modify the method described above slightly.

\section{RESULTS OF THE NUMERICAL COMPUTATIONS}

The methods described in Sections 4 and 5 were implemented and tested. (Some minor additions to the algorithms were made, for example, to look for degenerate solutions to Agler's equation where $A=0$ or $B=0$ and

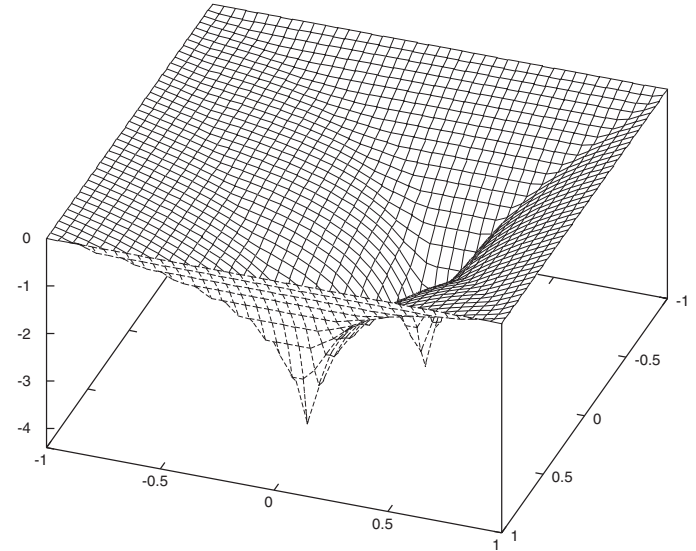

FIGURE 1. $\delta(z ; p+q)$, where $p=(0.6,0), q=(0,0.3)$, and $z \in \mathbb{D}^{2} \cap \mathbb{R}^{2}$.

some tweaks to obtain better convergence when $A$ or $B$ is "close" to 0.)

The program was run with 30,000 random choices of $p$ and $q$ and it was found that $\exp \delta$ and $\exp \delta^{*}$ differed by less than $3 \times 10^{-6}$ for all of these points. The program was also rerun with some parameters adjusted to improve the accuracy (sacrificing some computational speed, of course) with 1,000 random choices of $p$ and $q$. For all of these points, $\exp \delta$ and $\exp \delta^{*}$ differed by less than $3 \times 10^{-9}$. These computations suggest that $\delta=\delta^{*}$ for the bidisk with two poles of equal weight.

We also computed the values of $\delta$ and $\delta^{*}$ along some two-dimensional slices in order to draw some pictures. Figure 1 shows $\delta(z ; p+q)$ along a totally real twodimensional submanifold of $\mathbb{D}^{2}$ passing through $p$ and $q$. Figure 2 shows $\delta(z ; p+q)$ along an analytic disc through $p$.

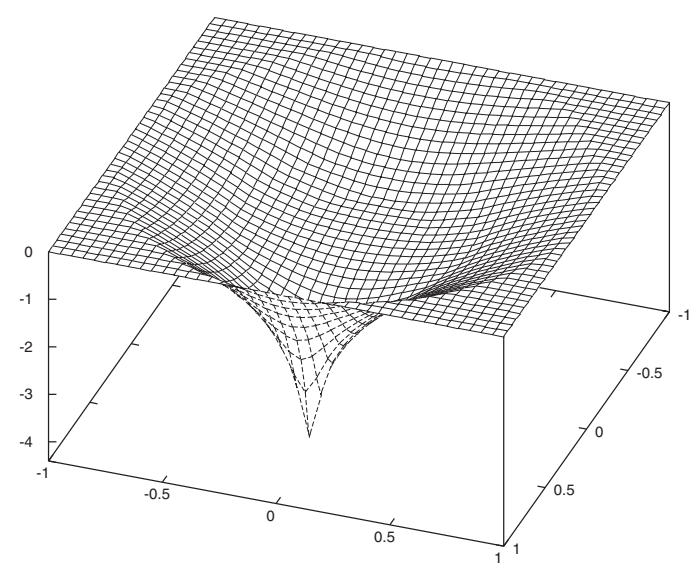

FIGURE 2. $\delta(z ; p+q)$, where $p=(0.6,0), q=(0,0.3)$, and $z \in \mathbb{D} \times\{0\}$. 


\section{A COUNTEREXAMPLE TO COMAN'S CONJECTURE}

We have seen that $\delta^{*}, g$, and $\delta$ have many properties in common, but nevertheless it turns out that Coman's conjecture fails in general. The first example of this is as mentioned in the introduction due to Carlehed and Wiegerinck [Carlehed and Wiegerinck 03] and concerns the case when $\Omega=\mathbb{D}^{2}$ and $\nu=w+\nu w^{\prime}$ with $w, w^{\prime} \in$ $\mathbb{D} \times\{0\}$ and $\nu \neq 1$. We will see that something similar is true when $\Omega=\mathbb{B}$ is the unit ball in $\mathbb{C}^{2}$, at least if the poles are sufficiently close together.

Theorem 7.1. Let $\mathbb{B}$ be the unit ball in $\mathbb{C}^{2}$ and let $w=0$, $w^{\prime}=(1 / 2,0)$. Then $\delta\left(\cdot ; w+2 w^{\prime}\right)$ is not plurisubharmonic on $\mathbb{B}$. In particular, $g\left(z ; w+2 w^{\prime}\right)<\delta\left(z ; w+2 w^{\prime}\right)$ for some $z \in \mathbb{B}$.

The proof of this theorem will be done by combining some traditional methods with numerical computation using interval arithmetic. Briefly put, interval arithmetic is a method of handling computations with real numbers on a computer in a way that gives stringent upper and lower bounds for all of the calculated variables. Even with the help of interval arithmetic, one can almost never hope to prove that two real numbers are equal with the help of a computer. On the other hand, by computing things with sufficiently high precision, it is sometimes possible to prove that two real numbers are not equal. For a more comprehensive introduction to interval arithmetic, we refer to [Moore 97].

Proof: Let $z=(0, c)$. First of all, let us find necessary and sufficient conditions on $\alpha$ and $\beta$ such that there exists an analytic disk $\phi: \mathbb{D} \rightarrow \mathbb{B}$ with $\phi(0)=z, \phi(\alpha)=w$, and $\phi(\beta)=w^{\prime}$.

Assume that $\phi$ is such a disk. Write $\phi=\left(\phi_{1}, \phi_{2}\right)$. Since $\phi_{1}(\alpha)=\phi_{2}(\alpha)=0$, we can factor the components of $\phi$ as

$$
\phi(\zeta)=\left(\phi_{1}(\zeta), \phi_{2}(\zeta)\right)=\frac{\alpha-\zeta}{1-\bar{\alpha} \zeta}\left(\psi_{1}(\zeta), \psi_{2}(\zeta)\right)
$$

and it follows from the maximum principle that $\psi=$ $\left(\psi_{1}, \psi_{2}\right): \mathbb{D} \rightarrow \mathbb{B}$. Using that $\delta$ is decreasing under holomorphic mappings, we have the following necessary conditions:

$$
\begin{aligned}
& \log |\alpha|=\delta_{\mathbb{D}}(0, \alpha) \geq \delta_{\mathbb{B}}(\phi(0), \phi(\alpha))=\delta_{\mathbb{B}}(z ; w), \\
& \log |\beta|=\delta_{\mathbb{D}}(0, \beta) \geq \delta_{\mathbb{B}}(\phi(0), \phi(\beta))=\delta_{\mathbb{B}}\left(z ; w^{\prime}\right),
\end{aligned}
$$

and similarly from $(7-1)$, we get

$\log |\beta|=\delta_{\mathbb{D}}(0, \beta) \geq \delta_{\mathbb{B}}(\psi(0), \psi(\beta))=\delta_{\mathbb{B}}\left(\frac{z}{\alpha} ; \frac{(1-\bar{\alpha} \beta) w^{\prime}}{\alpha-\beta}\right)$.

Conversely, if these conditions are satisfied, it is straightforward to see that such a disk $\phi$ exists.

Now, an explicit formula for $\delta_{\mathbb{B}}(z ; w)$ is of course well known (we have that $\delta_{\mathbb{B}}(z ; w)=\log \left|T_{w}(z)\right|$, where $T_{w} \in$ $\operatorname{Aut}(\mathbb{B})$ and $T_{w}(w)=0$ ). Our choice of $z, w$, and $w^{\prime}$ makes the resulting inequalities easier to handle than in the general setting. Untangling (7-2)-(7-4), we end up with

$$
\begin{aligned}
|\alpha| & \geq|c| \\
2|\beta| & \geq \sqrt{1+3|c|^{2}} \\
|\beta|^{2} & \geq 1-\left(1-\frac{1}{4}\left|\frac{1-\bar{\alpha} \beta}{\alpha-\beta}\right|^{2}\right)\left(1-\frac{|c|^{2}}{|\alpha|^{2}}\right) .
\end{aligned}
$$

We are interested in computing $\delta\left(z ; w+2 w^{\prime}\right)$, so we want to minimize $\log |\alpha|+2 \log |\beta|$ under these side conditions. To do this explicitly seems rather tricky, but using a numerical approach, we can compute $\delta$ to any desired accuracy and this will be enough to show that the function $c \mapsto \delta\left((0, c) ; w+2 w^{\prime}\right)$ is not subharmonic on $\mathbb{D}$. First note that (after a rotation) we can assume that $0<\alpha<1$. Also, the condition (7-6) only depends on $|\beta|$ and one can check that the right-hand side of $(7-7)$ is (for a fixed $0<\alpha<1$ and $|\beta|)$ smallest when $\beta$ is negative real. So we may as well assume that $\alpha=a$ and $\beta=-b$ with $0<a, b<1$. Furthermore, by biholomorphic invariance, we may also assume that $c$ is positive real. With these assumption, condition (7-7) simplifies to

$$
\left(a^{2} b^{2}-c^{2}\right)(a+b)^{2}-\frac{1}{4}\left(a^{2}-c^{2}\right)(1+a b)^{2} \geq 0 .
$$

The left-hand side of (7-8) is a quartic polynomial in $a$, say $p(a)$. Expanding this polynomial, we see that the coefficients of $a^{4}$ and $a^{3}$ are positive and the coefficients of $a^{1}$ and $a^{0}$ are negative. Hence, using Descarte's Rule of Signs, it follows that $p$ has at most one positive real zero. On the other hand, $p(c)=c^{2}\left(b^{2}-1\right)(c+b)^{2} \leq 0$ and $p(1)=(1+b)^{2}\left(b^{2}-1 / 4-3 c^{2} / 4\right) \geq 0$, so for a fixed $b$ and $c, p$ has exactly one zero in the interval $[c, 1]$. Hence, there is a unique $\tilde{a}(b) \geq c$ such that $(7-8)$ holds for all $a \geq \tilde{a}(b)$. Consequently, we want to minimize $\tilde{a}(b) b^{2}$ over all $b \leq 1$ satisfying $(7-6)$.

At this point, interval arithmetic comes in very handy. For fixed $c$ and $b$, it is easy to compute $\tilde{a}(b)$ with any desired accuracy (for example, using interval bisection or 
by solving the quartic equation explicitly). To find the minimum of $f(b)=\tilde{a}(b) b^{2}$, we can check (by implicit differentiation) that $f \in C^{1}$ so we only have to look for zeros of $f^{\prime}$ (and check the endpoints). Since we can compute $\tilde{a}(b)$ accurately, we can also compute $f^{\prime}$ accurately $\left(\tilde{a}^{\prime}(b)\right.$ can be expressed in terms of $\tilde{a}(b)$ and $b$ by differentiating the expression $p(\tilde{a}(b))=0)$. Using a so-called branch and bound algorithm, we can find good estimates for the zero(s) of $f^{\prime}$ and actually prove that $f^{\prime}$ has no other zeros. Doing all this, one can compute $\delta\left(z ; w+2 w^{\prime}\right)$ with any desired accuracy (let us stress the fact that the result of this computation is rigorous upper and lower bounds for $\delta$ ).

How does this information help us prove that $\delta$ fails to be plurisubharmonic? One approach could be to (numerically) compute the Laplacian of $c \mapsto \delta\left((0, c) ; w+2 w^{\prime}\right)$, but this is very difficult to do with good error estimates. A better way is to show that $\delta$ violates the submeanvalue property, since integration is far easier to do in a numerically rigorous way than differentiation. In our particular case, it is easy to check that the function $\delta(r)=\delta\left((0, r) ; w+2 w^{\prime}\right)$ is increasing in $r$ (when $0<r<1)$, since if $\phi=\left(\phi_{1}, \phi_{2}\right)$ is a disk in the defining family for $\delta(r)$ and $r^{\prime}<r$, then $\tilde{\phi}=\left(\phi_{1}, r^{\prime} r^{-1} \phi_{2}\right)$ is a member in the defining family for $\delta\left(r^{\prime}\right)$ and $d(\phi)=d(\tilde{\phi})$.

This monotonicity of $\delta(r)$ makes things even better. Let $z_{0}=\left(0, c_{0}\right) \in \mathbb{B}$ and $t=(0,1) \in \mathbb{C}^{2}$. To give lower and upper bounds for the integral

$$
\begin{aligned}
I & =\frac{1}{2 \pi} \int_{0}^{2 \pi} \delta\left(z_{0}+\varepsilon \exp (i \theta) t ; w+2 w^{\prime}\right) \mathrm{d} \theta \\
& =\frac{1}{\pi} \int_{0}^{\pi} \delta\left(z_{0}+\varepsilon \exp (i \theta) t ; w+2 w^{\prime}\right) \mathrm{d} \theta
\end{aligned}
$$

we divide $[0, \pi]$ into a suitable number of intervals, and use the fact that the integrand is decreasing in $\theta$. Hence, if $0=\theta_{0}<\theta_{1}<\cdots<\theta_{n}=\pi$ is a partition of $[0, \pi]$, then

$$
\sum_{j=1}^{n}\left(\theta_{j}-\theta_{j-1}\right) \underline{\delta}_{j}<I<\sum_{j=0}^{n-1}\left(\theta_{j+1}-\theta_{j}\right) \bar{\delta}_{j}
$$

where $\left[\underline{\delta}_{j}, \bar{\delta}_{j}\right]$ is an enclosure for $\delta\left(z_{0}+\varepsilon \exp \left(i \theta_{j}\right) t ; w+\right.$ $\left.2 w^{\prime}\right)$ obtained as described above. Performing these computations (and paying attention to rounding issues) with $c_{0}=0.3, \varepsilon=0.08$, and 128 subintervals of equal length, we obtain

$$
\delta\left(z_{0} ; w+2 w^{\prime}\right)>-1.29047>-1.29377>I,
$$

which proves that $\delta\left(\cdot ; w+2 w^{\prime}\right)$ is not plurisubharmonic.
Remark 7.2. Similar computations indicate that if $\nu>1$ and $w^{\prime}=(r, 0)$, then $\delta\left(\cdot ; 0+\nu w^{\prime}\right)$ is not plurisubharmonic if $r$ is sufficiently small. The results of these computations are shown in Table 1.

$\begin{array}{cccccc}\nu & r & c & \varepsilon & \delta & I \\ 2.0 & 0.5 & 0.3 & 0.12 & -1.29046 & -1.29388 \\ 1.9 & 0.5 & 0.3 & 0.12 & -1.26864 & -1.27083 \\ 1.8 & 0.5 & 0.3 & 0.12 & -1.24954 & -1.25089 \\ 1.7 & 0.5 & 0.3 & 0.12 & -1.23334 & -1.23411 \\ 1.6 & 0.5 & 0.3 & 0.12 & -1.22028 & -1.22066 \\ 1.5 & 0.5 & 0.3 & 0.12 & -1.21070 & -1.21084 \\ & & & & & \\ 1.4 & 0.3 & 0.22 & 0.06 & -1.57443 & -1.57522 \\ 1.3 & 0.3 & 0.22 & 0.06 & -1.54381 & -1.54411 \\ 1.2 & 0.3 & 0.22 & 0.06 & -1.52267 & -1.52273 \\ & & & & & \\ 1.1 & 0.15 & 0.22 & 0.06 & -1.53257 & -1.52382 \\ 1.05 & 0.15 & 0.22 & 0.06 & -1.51692 & -1.51694 \\ & & & & & \\ 1.03 & 0.10 & 0.15 & 0.06 & -1.899993 & -1.90003 \\ 1.02 & 0.10 & 0.15 & 0.06 & -1.897930 & -1.897937 \\ & & & & & \\ 1.01 & 0.08 & 0.10 & 0.04 & -2.302750 & -2.302751\end{array}$

TABLE 1. Computational results. $\delta=\delta((0, c) ; 0+\nu$. $(r, 0)) . I=(2 \pi)^{-1} \int_{0}^{2 \pi} \delta((c+\varepsilon \exp (i \theta), 0) ; 0+\nu \cdot(r, 0)) \mathrm{d} \theta$. The values for $\delta$ were computed by the interval method described above and are accurate to all digits given. The values of $I$ are numerically computed and should be accurate to all digits given. Furthermore, in all of the cases listed in the table except the last three, $I$ was also computed with rigorous bounds as described in the proof of Theorem 7.1 to a sufficiently high accuracy to ensure that the submeanvalue property was violated.

\section{ACKNOWLEDGMENTS}

This work was supported by grants from the Royal Swedish Academy of Sciences and the European Union Research Training Network Operators and Analysis.

\section{REFERENCES}

[Agler 98] J. Agler. "Some Interpolation Theorems of Nevanlinna-Pick Type." Preprint, 1998.

[Agler and McCarthy 99] J. Agler and J. E. McCarthy. "Nevanlinna-Pick Interpolation of the Bidisk." J. Reine Angew. Math. 506 (1999), 191-204.

[Bedford and Taylor 76] E. Bedford and B. A. Taylor. "The Dirichlet Problem for a Complex Monge-Ampère Equation." Invent. Math. 37 (1976), 1-44. 
[Błocki 99] Z. Blocki. "The Complex Monge-Ampère Operator in Pluripotential Theory." Preprint, 1999.

[Carlehed 95] M. Carlehed. "Some Properties of the Pluricomplex Green Function and Potentials." Research Reports No. 14, Department of Mathematics, Umeå University, 1995.

[Carlehed and Wiegerinck 03] M. Carlehed and J. Wiegerinck. "Le cône des functions plurisousharmoniques negatives et une conjecture de Coman." Ann. Polon. Math. 80 (2003), 93-108.

[Coman 00] D. Coman. "The Pluricomplex Green Function with Two Poles of the Unit Ball of $C^{n}$." Pacific J. Math. 194 (2000), 257-283.

[Demailly 87] J. -P. Demailly. "Mesures de Monge-Ampère et measures pluriharmoniques." Math. Z. 194 (1987), 519564.

[Edigarian and Zwonek 98] A. Edigarian and W. Zwonek. "Invariance of the Pluricomplex Green Function under Proper Mappings with Applications." Complex Variables Theory Appl. 35 (1998), 367-380.

[Klimek 85] M. Klimek. "Extremal Plurisubharmonic Functions and Invariant Pseudodistances." Bull. Soc. Math. France 113 (1985), 231-240.
[Klimek 91] M. Klimek. Pluripotential Theory. New York: The Clarendon Press-Oxford University Press, 1991.

[Lelong 89] P. Lelong. "Fonction de Green pluricomplexe et lemmes de Schwarz dans les espaces de Banach." J. Math. Pures Appl. 9:68 (1989), 319-347.

[Lempert 81] L. Lempert. "La métrique de Kobayashi et la representation des domains sur la boule." Bull. Soc. Math. France 109 (1981), 427-474.

[Moore 97] R. E. Moore. Interval Analysis. Englewood Cliffs, NJ: Prentice-Hall, 1997.

[Pick 16] G. Pick. "Über eine Eigenschaft der konformen Abbildung kreisförmiger Bereiche." Math. Ann. 77 (1916), 7-23.

[Thomas and Trao 03] P. Thomas and N. V. Trao. "Pluricomplex Green and Lempert Functions for Equally Weighted Poles." Ark. Mat. 41 (2003), 381-400.

[Wikström 99] F. Wikström. "Non-linearity of the Pluricomplex Green Function." Proc. Amer. Math. Soc. 129 (2001), 1051-1056.

Frank Wikström, Department of Mathematics, Mid Sweden University, 85730 Sundsvall, Sweden (Frank.Wikstrom@mh.se)

Received April 25, 2003; accepted September 9, 2003. 